\title{
Role of transnasal endoscopic surgery for the management of orbital diseases
}

\author{
Susan A Thomas ${ }^{1}{ }^{\oplus}$, Suresh Pillai ${ }^{2}$, Sanu P Moideen ${ }^{1}[0$ \\ ${ }^{1}$ Department of Otorhinolaryngology - Head and Neck Surgery, MOSC Medical College, Kolenchery, \\ Ernakulam, India \\ ${ }^{2}$ Department of Otorhinolaryngology - Head and Neck Surgery, KMC Manipal, Karnataka, India
}

\begin{abstract}
BACKGROUND. Indications for endoscopic sinus surgery have been widely expanding since its introduction, to such an extent that it is now accepted as the ideal method for the treatment of some orbital conditions. Endoscopic approach to orbits provides excellent visualization with minimal cosmetic and functional morbidity.

MATERIAL AND METHODS. This descriptive study has been conducted in the Department of Otolaryngology - Head and Neck Surgery, KMC, Manipal, during July 2010 and July 2014. We evaluated the role of endoscopic sinus surgery (ESS) as a surgical mode of treatment in different types of orbital diseases.

RESULTS. 24 patients underwent ESS approach for various ophthalmologic conditions.

CONCLUSION. Results from our studies showed that the endoscopic sinus surgery approach is effective and has better results than an open approach in the management of certain types of orbital pathology.
\end{abstract}

KEYWORDS: endoscopic sinus surgery, orbit surgery, optic nerve, FESS, ESS.

\section{INTRODUCTION}

Since functional endoscopic sinus surgery (FESS) was introduced in 1985 by the brilliant work of Messerklinger and further popularized by Stammberger, it has become the standard for surgical treatment of chronic rhinosinusitis ${ }^{1}$. Works published by Kennedy et al. in 1990 expanded the indication for endoscopic sinus surgery (ESS) to the treatment of certain orbital diseases ${ }^{2}$.

With the advancement of ESS, not only sinonasal causes of orbital pathology but also isolated primary orbital conditions, such as orbital injuries and neoplasms, are now treated by endoscopic approach.

\section{AIM AND OBJECTIVES}

This study was conducted in order to evaluate the role of the ESS approach as a surgical mode of treatment in orbital diseases.
Objectives of the study were to understand the clinico-pathological profile, clinical examination, radiological and intraoperative findings, treatment outcomes in patients who underwent ESS for orbital pathologies.

\section{MATERIAL AND METHODS}

\section{Study setting}

This descriptive study was conducted in the Department of Otolaryngology - Head and Neck Surgery, KMC, Manipal, during July 2010 and July 2014.

\section{Patients' selection}

Those patients who underwent ESS for orbital pathologies during the study period were included in the study.

Patients who have undergone ESS which have no therapeutic or diagnostic value for their orbital pathology were excluded from the study. 


\section{Methodology}

Prior to the ESS, patient data was collected using an institutional Ethical Committee approved proforma. Preoperative ophthalmological evaluation and a plain computed tomography (CT) scan of the nose, the paranasal sinuses and the orbit was performed in all cases. Those who were suspected to have intracranial complication underwent contrast enhanced brain CT and /or MRI and neurological evaluation.

A diagnostic nasal endoscopy (DNE) was done preoperatively. Mucopus or allergic mucin or fungal debris, if present, was collected using a nasal swab and sent for culture. In cases with a suspicion of neoplasia or invasive fungal sinusitis, biopsy was taken for histopathological examination.

After completing other routine and relevant blood investigations prior to surgery, patients were taken for ESS with a written informed consent for endoscopic approach. The consent for an open approach, if needed, was also taken in all cases.

All the patients underwent ESS, the extent decided as per the nature of each patient's disease. During the surgery the goals set were:

1) Diagnostic aid by culture \& sensitivity (C\&S) / fungal culture/ tissue biopsy.

2) Drainage, decompression / debridement of therapeutic intent.

Postoperative nasal packing was done for all cases, except those patients in whom orbital and optic decompression was done. On postoperative day one (POD1), endoscopic cleaning was performed to remove debris along with pack removal, and patients were started on saline irrigations and local steroid spray. Patients also received an oph- thalmology evaluation. Systemic antifungals, like fluconazole or itraconazole, were started for all fungal culture positive cases. Patients were discharged when they were symptomatically better and fit for discharge. All patients received a minimum of three postoperative endoscopic nasal cleaning, excluding the one on POD1.

Upon discharge, all patients were followed up in the $1^{\text {st }}$ week, $3^{\text {rd }}$ week, $3^{\text {rd }}$ and $6^{\text {th }}$ months.

Data collected in the preoperative, postoperative and follow-up period via the proforma were then put together as the Master chart and was analysed using SPSS software.

\section{RESULTS}

A total of 24 patients underwent the ESS approach for management of diseases involving the orbit during the study period.

Among the patients' clinical profile, 12 patients were diagnosed with orbital cellulitis and subperiosteal abscess, 6 patients had fungal rhinosinusitis with orbital extension and 2 patients had sino-orbital cemento-ossifying fibroma. Other diagnoses, such as frontal mucocele, sino-orbital transitional cell carcinoma, sino-orbital inflammatory myoblastic tumour, orbital subperiosteal hematoma, were seen in one patient each. These results are tabulated in Table 1.

\section{Orbital cellulitis and subperiosteal abscess}

A total of 12 patients underwent ESS as part of the treatment for orbital cellulitis and subperiosteal abscess. Their age ranged between $3-55$ years

Table 1. Clinical conditions of the patients included in our study group.

\begin{tabular}{cc}
\hline Pathology & Number of patients \\
\hline Orbital cellulitis and subperiosteal abscess & 12 \\
\hline Fungal rhinosinusitis with orbital extension & 6 \\
\hline Sino-orbital cemento-ossifying fibroma & 2 \\
\hline Frontal mucocele & 1 \\
\hline Sino-orbital transitional cell carcinoma & 1 \\
\hline Sino-orbital inflammatory myoblastic tumour & 1 \\
\hline Orbital subperiosteal hematoma & 1
\end{tabular}




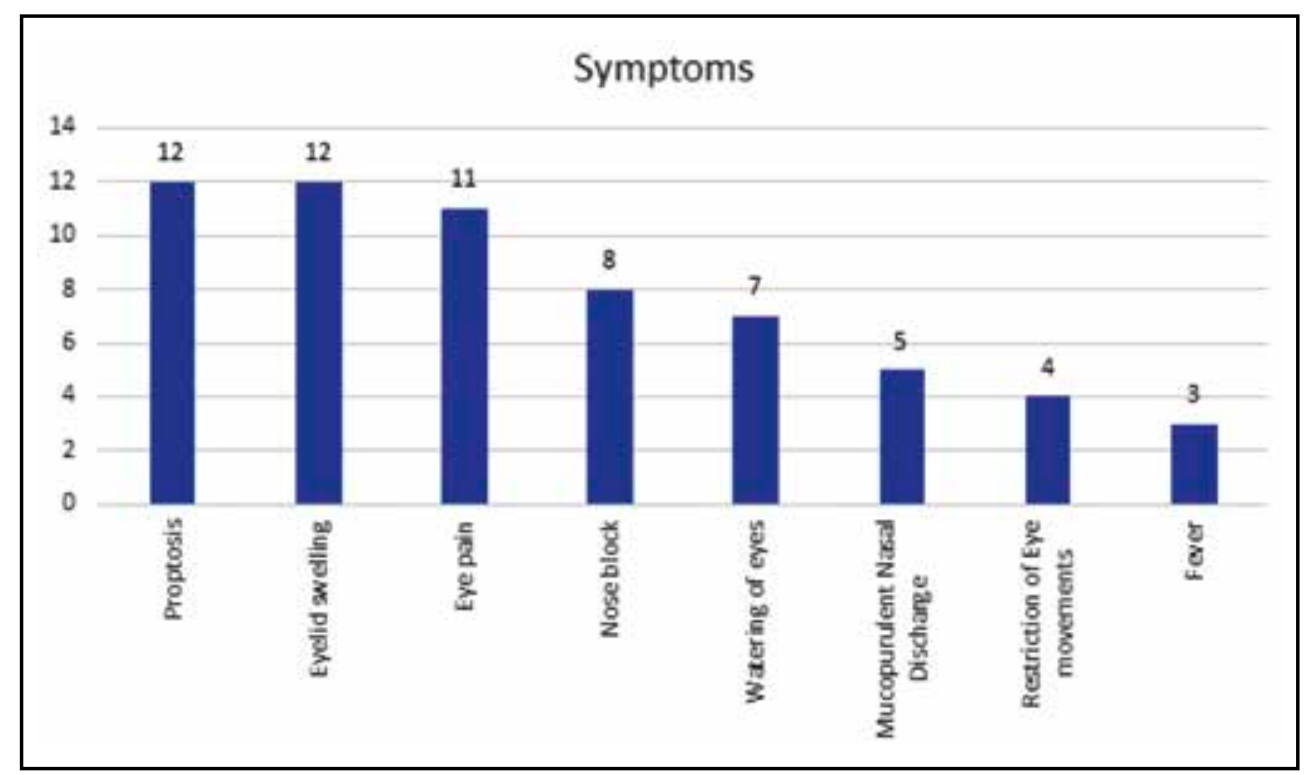

Figure 1. Symptomatology in patients with orbital cellulitis and subperiosteal abscess.

(with a mean age of 29.6 years), of which 2 were paediatric patients and 10 were adults, with an average duration of 6.3 days (2 - 21 days). The most common symptoms were proptosis and eyelid swelling (present in all patients), followed by eye pain (11 patients), nasal block (8 patients), mucopurulent nasal discharge (5 patients). 4 patients had restriction of eye movements and $3 \mathrm{had}$ fever at the time of presentation. These symptoms are represented in Figure 1.

Out of 12 patients, 10 had acute rhinosinusitis and 2 patients had acute dacryocystitis with lacrimal abscess. Two patients had diabetes mellitus as a risk factor. One rhinosinusitis patient had given history of nasal surgery, while another gave history of septal trauma.

In the preoperatory imaging studies, 10 patients had unilateral sinus disease, while 2 patients had no sinus disease. Among the sinuses involved, the frontal and ethmoid sinuses were involved in 10 patients, the maxillary sinus in 9 patients and the sphenoid sinus in 8 patients. Coming to the orbital findings, proptosis and orbital fat stranding were present in all 12 patients. 3 patients had orbital subperiosteal abscess, 2 had lacrimal sac abscess and one patient had dehiscence of the lamina papyracea.

Intraoperatively, pus was drained on elevation of the lamina papyracea in 8 out of 12 patients, indicating subperiosteal abscess. One patient had frontal extradural abscess at the time of presentation.

Endoscopic approach alone was enough in 7 patients, while 5 patients needed combined endoscopic - external approach.

Histopathology reports of 4 cases showed evi- dence of chronic rhinosinusitis. Bacterial growth was observed in the culture of mucopus from 4 out of 12 patients. Methicillin-resistant Staphylococcus aureus, Methicillin-sensitive Staphylococcus aureus, Coagulase-negative Staphylococcus, Burkholderia cepacia were seen in one patient each.

There was complete recovery in terms of visual improvement, subsidence of eye pain, watering of eyes, proptosis and restriction of eye movements comparable to disease prior state in all patients.

\section{Fungal rhinosinusitis}

6 patients presented with orbital complications of fungal rhinosinusitis with an age ranging between 1 year to 80 years. Gender ratio observed as male to female ratio was $1: 1$.

Out of the 6 patients, headache, decreased vision and eye pain was common among all five patients, except for the child. Other symptoms in patients with fungal rhinosinusitis were proptosis (4 patients), impaired color vision (4 patients), fever (3 patients) and mucopurulent nasal discharge (1 patient). The symptomatology of these patients is listed in Figure 2.

Preoperatively, DNE showed unilateral mucopurulent nasal discharge in the middle meatus and the spheno-ethmoidal recess in four out of six cases. On ophthalmological evaluation, visual impairment or loss of perception of light was present in 2 patients, counting of fingers at 1 meter was affected in 2 patients and vision was normal in one patient. Visual assessment could not be done in one patient as it was a 1-year-old baby. Restriction of eye movements or absent movements were ob- 


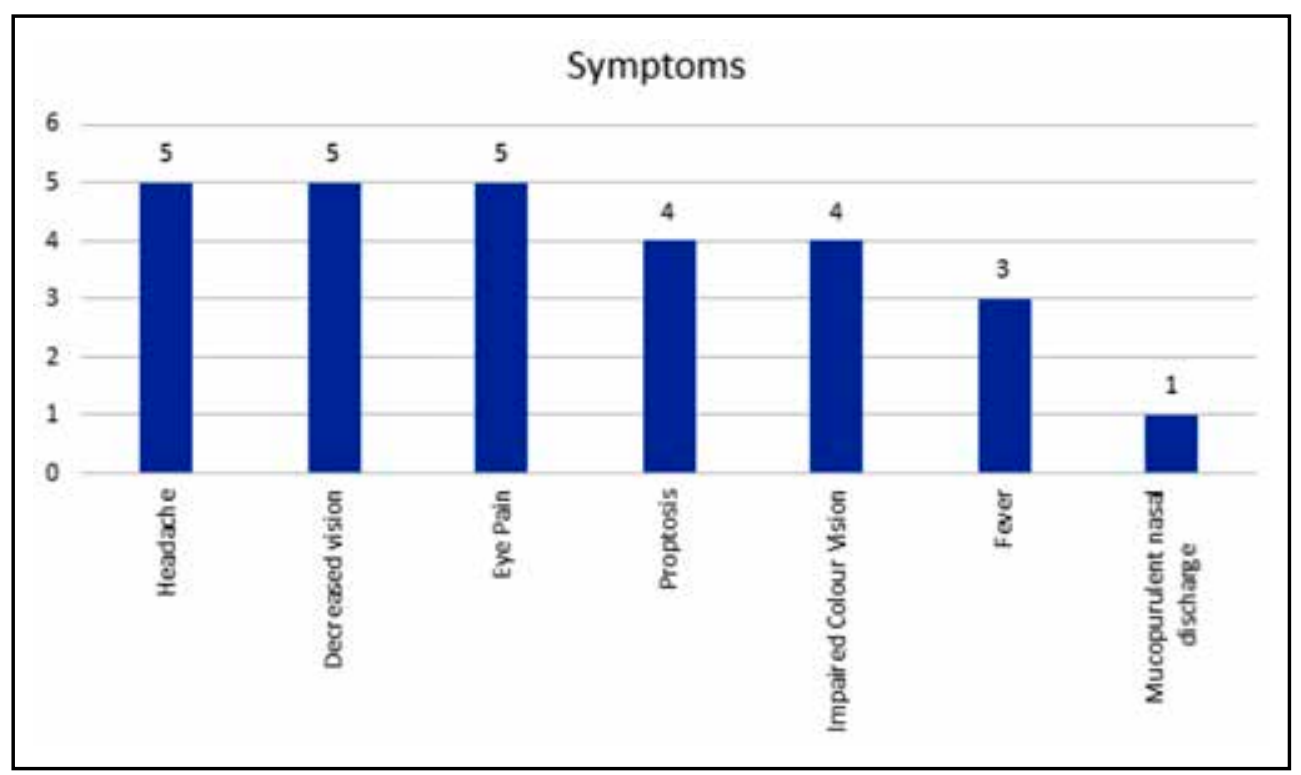

Figure 2. Symptomatology of the patients with fungal rhinosinusitis.

served in two people, partial movements in 3 people and it was normal in one patient. Corneal opacity was noted in the child.

Diabetes mellitus (4 patients), immunocompromising conditions (3 patients) and developmental delay (the child) were the comorbidities present in patients diagnosed with fungal rhinosinusitis.

Histopathological examination and fungal culture showed candida osteomyelitis in the paediatric patient, mucormycosis in 2 patients, aspergillosis in one patient and no fungus in 2 patients.

The surgical approach was endoscopic in all six cases, five of the cases with therapeutic intention and one case with diagnostic intention alone.

Mortality was 2 out of 6 , visual deterioration was observed in one case of invasive fungal sinusitis when compared to the pre-disease state, and no improvement in vision was noted in surviving invasive fungal sinusitis cases. There was complete recovery of visual and orbital symptoms of allergic fungal sinusitis.

\section{Sino-orbital cemento-ossifying fibroma}

Two boys of ages 10 and 12, with cemento-ossifying fibroma underwent orbital surgery in the study period. Both presented with proptosis and reduced vision. History of previous optic nerve decompression and craniotomy was present for the 12-yearold boy.

Radiological studies showed intracranial extension in one case and unilateral posterior ethmoids involvement, abutting the optic nerve in the other case (Figure 3).

Repeated optic nerve decompression and craniofacial resection for the residual tumour was done for one case, but the child was lost to followup. Endoscopic partial excision (abutting the optic nerve, preservation of vision) was done for the second child and he is still on follow-up for 6 months without any symptoms.

\section{Sino-orbital transitional cell carcinoma}

An 80-year-old female presented with complaints of absent vision and eye movements in the right eye. On examination, proptosis, a frozen eye with absent perception of light in the right eye was noted and orbital apex syndrome was diagnosed.

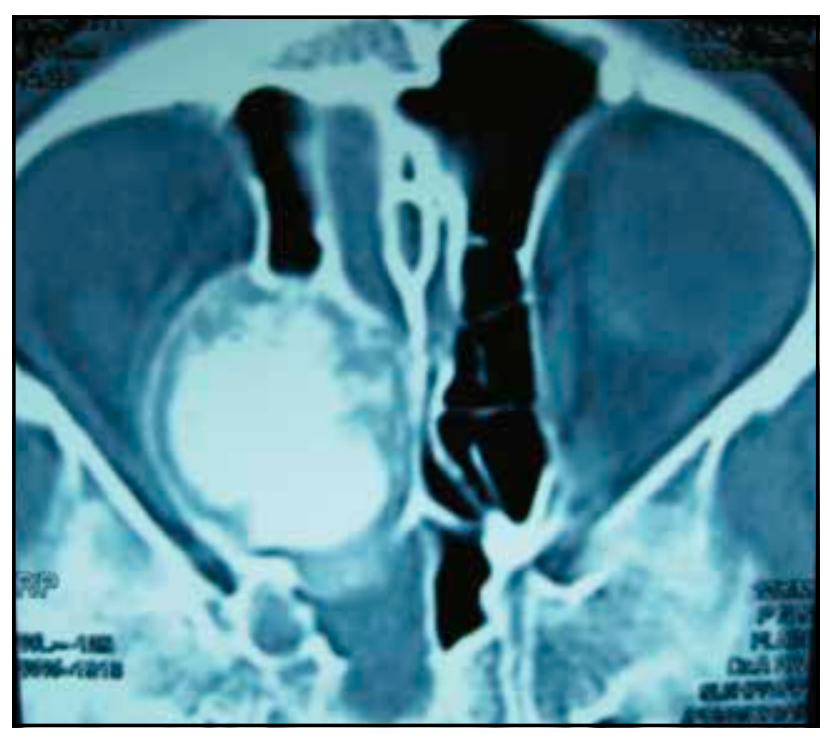

Figure 3. Cranio-facial, axial CT image showing cemento-ossifying fibroma involving the right orbit, abutting the optic nerve. 

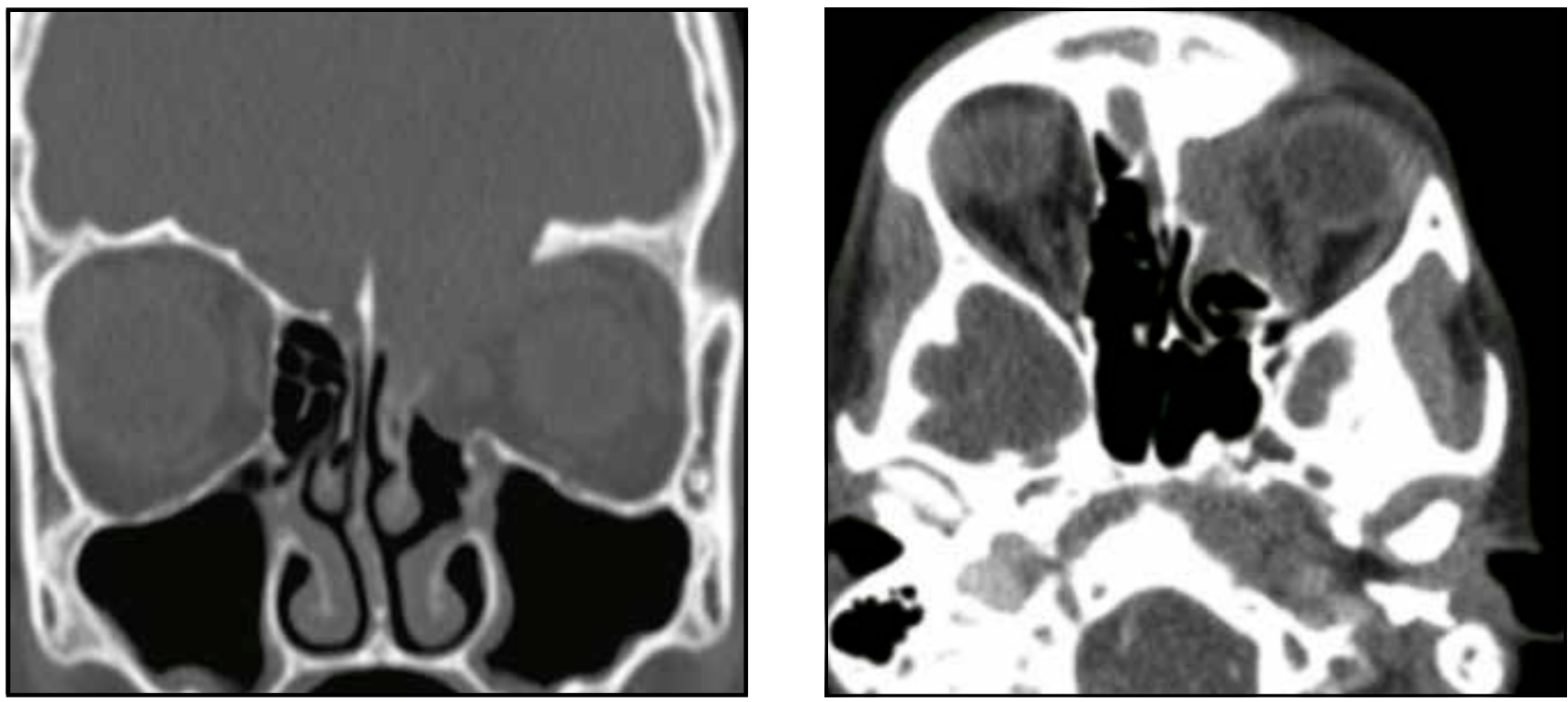

Figure 4. Cranio-facial CT imaging (coronal and axial slices) of inflammatory myofibroblastic tumor with intracranial extension.

The CT scan of the orbit and paranasal sinuses showed a contrast enhancing mass in the right posterior sinuses with extension into the orbital apex.

ESS with endoscopic biopsy from the orbit and posterior sinuses was done.

Patient was lost to follow-up after histopathological diagnosis of transitional cell carcinoma.

\section{Frontal mucocele}

A single case of a 56-year-old female presented with complaints of proptosis, frontal headache for 2 months. She was a known case of diabetes mellitus and hypertension. DNE had no features of osteomeatal disease. The CT scan of the paranasal sinuses showed a right frontal mucocele.

She underwent endoscopic marsupialisation of the frontal mucocele. There was complete recovery following the treatment.

\section{Sino-orbital inflammatory myoblastic tumour}

A 14-year-old boy presented with complaints of left frontal headache, proptosis and eye pain. The cranio-facial contrast enhancing CT (CECT) scan showed a contrast enhancing mass in the left posterior ethmoid and sphenoid with erosion of lamina papyracea and extraconal involvement of the orbit (Figure 4). DNE and biopsy were done and were reported as inconclusive. Endoscopic endonasal resection of the mass was done and sent for histopathological examination. It was reported as an inflammatory myoblastic tumour. The patient was on regular monthly follow-up.

Sixth month after the surgery, the patient presented with seizures and the brain CECT revealed a contrast enhancing mass in the posterior ethmoid, orbit, with intracranial extension. Frontal craniotomy and complete resection of the tumour was done. The patient is now on regular follow-up for 6 months without recurrence.

\section{Orbital subperiosteal hematoma}

A 12-year-old male presented with post-traumatic eye swelling and pain on the right side. The child had no visual disturbance. The CT scan of the orbit showed a subperiosteal hematoma involving the medial and lateral wall of the orbit.

Endoscopic and external drainage was done for the child, following which complete recovery was observed.

\section{DISCUSSIONS}

Endoscopic sinus surgery has two major roles in the management of orbital diseases. One is for the diagnosis and the other is for the treatment of orbital pathology.

Kai-Li Liang et al. ${ }^{3}$ has described orbital complications secondary to bacterial rhinosinusitis, fungal rhinosinusitis, skull base osteomyelitis and mucoceles, subperiosteal hematoma, Graves' disease, traumatic optic neuropathy and orbital blowout fracture, as the various indications for which they have employed ESS. Over the years, many authors have added on to the list of indications for endoscopic endonasal approach to the orbit, like resection of orbital cavernous haemangioma, management of benign tumours located at the orbital 
apex, resection of orbital extension of the sinonasal tumours, etc.

In our study, the indications for endonasal endoscopic approach to the orbit were orbital cellulitis and subperiosteal abscess, orbital subperiosteal hematoma, sino-orbital candida osteomyelitis, sino-orbital invasive fungal sinusitis, allergic fungal sinusitis with orbital extension, sino-orbital transitional cell carcinoma for biopsy, sino-orbital inflammatory myoblastic tumour and sino-orbital cemento-ossifying fibroma.

For the management of orbital cellulitis and subperiosteal abscess that did not respond adequately to antibiotics, we have employed the endoscopic surgical approach alone in 7 patients. Combined endoscopic - external approach was employed in 2 cases of lacrimal abscess and 3 cases with associated lid abscess. Worsening of the ophthalmological function despite medical management is an indication for surgical intervention ${ }^{4,5}$.

Regarding the management of sino-orbital fungal rhinosinusitis, endoscopic sinus debridement slows the progression of the disease, reduces the fungal load and provides a specimen for culture and histopathologic diagnosis with limited morbidities ${ }^{6,7}$. In our cases, the surgical approach was endoscopic in all 5 cases -4 cases with therapeutic intention and one case (candida osteomyelitis) with diagnostic intention alone. All cases were treated with appropriate antifungals for the time prescribed by the physician after endoscopic resection.

Endoscopic marsupialization techniques are effective for the management of most cases of mucoceles $^{8}$. Our patient with frontal mucocele underwent an endoscopic approach.

Open surgical resection carries a high morbidity in the case of cemento-ossifying fibroma, given the complex anatomy and critical neurovascular structures that occupy the orbital apex. Endoscopic optic nerve decompression for benign tumours involving the orbit is effective for improvement in visual function, as measured by visual acuity, visual fields, and, in some cases, color vision ${ }^{9,10}$. In our study, there was also improvement in vision of the patient following decompression of the optic nerve.

Inflammatory myoblastic tumours have a predilection for children and adolescents, although they may arise as late as the eighth decade of life ${ }^{10}$. Complete surgical resection is described as the treatment of choice for these tumours. Recurrence can be high if the tumour is left behind as it is locally aggressive. There is no distant metastasis reported. In our case, with the endoscopic approach, there was recurrence of the tumour of the sphenoethmoid orbital complex, after 6 months, with intracranial extension. The patient had to undergo an open craniotomy approach to resect the intracranial extension of the tumour. At the 8-month follow-up the patient was asymptomatic without any clinical or endoscopic evidence of recurrence. Literature on this condition is limited for comparing the endoscopic approach versus the open approach for clearance of the disease.

Acute orbital haemorrhage may be a visual emergency, as vision can be promptly and irreversibly lost $^{11}$. In our study, we employed a combined approach as the hematoma was also involving the lateral wall of the orbit. There was complete remission of symptoms of proptosis in two days.

\section{CONCLUSIONS}

The transnasal endoscopic approach gives a better panoramic view with increased illumination and magnifications. Such visualization provides a potential for more complete disease removal with less impact on eye function and very low rate of complications. The transnasal endoscopic surgical technique for orbital and sino-orbital lesions is a minimally invasive alternative to traditional invasive approaches for selected patients and can achieve favourable outcomes with very low morbidity and less cosmetic compromise.

The orbital and sino-orbital lesions were managed via an external fronto-ethmoidectomy and lateral rhinotomy or even craniofacial approach, which allow thorough exposure and excellent access, but carry high postoperative morbidity.

The orbital diseases for which we undertook endoscopic endonasal approach included orbital complications secondary to bacterial rhinosinusitis, fungal rhinosinusitis and mucoceles, subperiosteal hematoma, neoplastic conditions like cemento-ossifying fibroma, inflammatory myoblastic tumour and sinonasal transitional cell carcinoma. Our results showed that ESS is effective and better than an open approach in the management of some of these orbital diseases.

\section{Acknowledgements. Nil}

Conflict of interest. The authors have no conflict of interest.

Contribution of authors. All authors have equally contributed to this work.

Ethical approval. All procedures performed in the study involving human participants were in accordance with the ethical standards of the institutional and/or national research committee and with the 1964 Helsinki declaration and its later amendments or comparable ethical standards. 


\section{REFERENCES}

1. Stammberger H, Posawetz W. Functional endoscopic sinus surgery. Concept, indications and results of the Messerklinger technique. Eur Arch Otorhinolaryngol. 1990;247(2):63-76. DOI: 10.1007/ BF00183169.

2. Kennedy DW, Goodstein ML, Miller NR, Zinreich SJ. Endoscopic transnasal orbital decompression. Arch Otolaryngol Head Neck Surg. $\quad 1990 ; 116(3): 275-82$. DOI: $10.1001 / \mathrm{ar}$ chotol.1990.01870030039006.

3. Liang KL, Su MC, Shiao JY, Hsin CH, Jiang RS. Endoscopic sinus surgery for the management of orbital diseases. ORL J Otorhinolaryngol Relat Spec. 2008;70(2):134-40. DOI: 10.1159/000114538.

4. Presutti L, Lucidi D, Spagnolo F, Molinari G, Piccinini S, AlicandriCiufelli M. Surgical multidisciplinary approach of orbital complications of sinonasal inflammatory disorders. Acta Otorhinolaryngol Ital. 2021;41 (2 Suppl 1):S108-15. DOI: 10.14639/0392-100Xsupp1.1-41-2021-11.

5. Al-Hassani JMK. Outcome of orbital complications of acute rhinosinusitis in children. Iraqi JMS. 2021;19(1):90-8. DOI: 10.22578/ IJMS.19.12.

6. Vengerovich G, Echanique KA, Park KW, Wells C, Suh JD, Lee JT, et al. Retrospective analysis of patients with acute invasive fungal rhi- nosinusitis in a single tertiary academic medical center: a 10-year experience. Am J Rhinol Allergy. 2020;34(3):324-30. DOI $10.1177 / 1945892419896233$.

7. Gür H, İsmi O, Vayısoğlu Y, Görür K, Arpacı RB, Horasan EȘ, et al. Clinical and surgical factors affecting the prognosis and survival rates in patients with mucormycosis. Eur Arch Otorhinolaryngol. 2021. DOI: 10.1007/s00405-021-06910-6. [Epub ahead of print].

8. Plantier DB, Neto DB, Pinna FR, Voegels RL. Mucocele: clinical characteristics and outcomes in 46 operated patients. Int Arch Otorhinolaryngol. 2019;23(1):88-91. DOI: 10.1055/s-0038-1668126.

9. Elkholy TA, Kamel RM, Bendary BA, Abosaf AI, Maqsoud RRA, Galal HM. Juvenile ossifying fibroma: multiportal endoscopic trans-nasal and orbital surgical approaches, histopathological and radiological findings. AIMJ. 2021;2(6):48-54. DOI: $10.21608 /$ aimj.2021.73978.1464

10. Strianese D, Tranfa F, Finelli M, Iuliano A, Staibano S, Mariniello G. Inflammatory myofibroblastic tumor of the orbit: A clinico-pathological study of 25 cases. Saudi J Ophthalmol. 2018;32(1):33-9. DOI: 10.1016/j.sjopt.2018.04.001.

11. Hashim SE, Fatisha A, Nazri MN. Managing subacute subperiosteal hematoma with high intraocular pressure: a case report. IIUM Medical Journal Malaysia. 2017;16(1)135-8. DOI: 10.31436/imjm. v16i1.371.

This is an open access article published under the terms and conditions of the Creative Commons Attribution-NonCommercial-NoDerivatives 4.0 International (CC BY-NC-ND 4.0) License (https://creativecommons.org/licenses/by-nc-nd/4.0/). CC BY-NC-ND 4.0 license requires that reusers give credit to the creator by citing or quoting the original work. It allows reusers to copy, share, read, download, print, redistribute the material in any medium or format, or to link to the full texts of the articles, for non-commercial purposes only. If others remix, adapt, or build upon the material, they may not distribute the modified material. 